\title{
Inhibition of Carrageenan/Kaolin-Induced Arthritis in Rats and of Inflammatory Cytokine Expressions in Human IL-1 $\beta$-Stimulated Fibroblast-like Synoviocytes by a Benzylideneacetophenone Derivative
}

\author{
Bongjun Sur, ${ }^{1}$ Seungmin Kang, ${ }^{1}$ Mijin Kim, ${ }^{1}$ and Seikwan Oh ${ }^{1,2}$
}

\begin{abstract}
The benzylideneacetophenone derivative JC3 [(2E)-3-(4-hydroxy-3-methoxyphenyl)phenylpro-2-en-1-one] (JC3) was synthesized by modifying yakuchinone B obtained from the seeds of Alpinia oxyphylla, a member of the ginger family (Zingiberaceae), which are widely used as a folk remedy and as an anti-inflammatory. The aim of this study was to investigate the anti-arthritic effects of $\mathrm{JC} 3$ in rat models of carrageenan-induced paw pain and carrageenan/kaolin-induced knee arthritis. The anti-nociceptive effect of JC3 was assessed by measuring paw withdrawal pressure thresholds using an analgesy-meter. Arthritic symptoms in our monoarthritic rat model were evaluated using weight distribution ratios (WDR), paw thicknesses, and serum prostaglandin E2 (PGE2), tumor necrosis factor (TNF)- $\alpha$, interleukin (IL)-6, and vascular endothelial growth factor (VEGF) levels (determined by ELISA). Histological analyses of knee joints were performed after injecting JC3 intraperitoneally into rats before carrageenan treatment at 5 or $10 \mathrm{mg} / \mathrm{kg} /$ day for 6 days. The anti-inflammatory effects of JC3 were investigated in vitro using interleukin-1beta (IL-1 $\beta$ )-stimulated fibroblastlike synoviocytes (FLS) derived from arthritis patients. PGE2, IL-6, and IL-8 levels were measured after treating FLS with JC3. In arthritis-induced rats, JC3 treatment significantly decreased nociceptive and arthritic symptoms at days 5 to 6 after carrageenan/kaolin injection. Histological staining of knee tissue showed that JC3 significantly reduced inflammatory areas in the knee joints. Furthermore, JC3 inhibited the expressions of IL-6 and IL-8 in FLS cells at concentrations of 5-10 $\mu \mathrm{g} / \mathrm{ml}$ and decreased $\mathrm{PGE}_{2}$ levels in FLS cells. These findings suggest JC3 has anti-arthritic effects in in vivo and in vitro, and that it might be useful for the treatment of arthritis.
\end{abstract}

KEY WORDS: Arthritis; Benzylideneacetophenone derivative; Anti-inflammatory; Fibroblast-like synoviocytes.

\footnotetext{
${ }^{1}$ Department of Molecular Medicine and TIDRC, School of Medicine, Ewha Womans University, Seoul, Republic of Korea

${ }^{2}$ To whom correspondence should be addressed at Department of Molecular Medicine and TIDRC, School of Medicine, Ewha Womans University, Seoul, Republic of Korea. E-mail: skoh@ewha.ac.kr
}

\section{INTRODUCTION}

Rheumatoid arthritis (RA) is a response of joint tissues to joint deformation, trauma or biochemical, genetic, or intracellular factors $[2,11]$. In arthritis, proliferative fibroblast-like synoviocytes (FLSs) play key roles in joint damage and in the propagation of inflammation because they produce considerable amounts of 
proinflammatory mediators, such as interleukin-6 (IL-6) and prostaglandin E2 (PGE2) [16]. RA progression is associated with increased cytokine levels of IL- $1 \beta$, which is produced by macrophages and dendritic cells [20]. Because IL-1 $\beta$ is believed to play a crucial role in synovial inflammation, elevating IL- $1 \beta$ levels in FLSs has been used to mimic the synovial proliferation that occurs in RA [13]. IL-1 $\beta$ is also known to prompt the over expressions of proinflammatory factors in many cell types [3]. IL-1 $\beta$ is an influential inducer of metalloproteinase production by FLSs [6]. The nonsteroidal anti-inflammatory agents used to treat RA are known to have harmful effects, particularly in the gastrointestinal tract, and thus, there is a need for anti-inflammatory drugs with fewer side effects $[8,12]$.

Yakuchinone B is a constituent of the seeds of Alpinia oxyphylla and is a conjugated 1,4-enone containing a phenyl ring, which is a feature of an important class of natural chalcones with wide-ranging biological activities [24]. Furthermore, yakuchinone B is known to have significant biological effects, which include antitumor [5], antiviral [17], and anti-inflammatory [21] effects. The benzylideneacetophenone JC3 was synthesized by modifying yakuchinone $\mathrm{B}$ in an effort to develop anti-inflammatory agents [18].

No previous study has been undertaken to investigate the anti-arthritic effects of JC3 on human FLS cells or in an animal model of arthritis. In this study, the antiarthritic effects of JC3 were evaluated in carrageenan/ kaolin-induced rat models and in IL- $1 \beta$-stimulated rheumatoid arthritis fibroblast-like synoviocytes derived from arthritis patients.

\section{MATERIALS AND METHODS}

\section{Isolation and Culture of FLSs}

From the RA patients who previously had joint replacement surgery, their synovial tissues were used to separate the fibroblast-like synoviocytes (FLSs), which are type of arthritic cells with active inflammation, which then were used to analyze the antiarthritic effect, as described previously [14]. Cells were grown in Dulbecco's modified Eagle medium (low glucose; Gibco-Invitrogen, Carlsbad, CA, USA) supplemented with $10 \%$ (vol/vol) fetal bovine serum (Gibco-Invitrogen), $100 \mathrm{U} / \mathrm{mL}$ penicillin, and $100 \mu \mathrm{g} / \mathrm{mL}$ streptomycin sulfate (GibcoInvitrogen). FLSs of passages 3-6 were used in the experiments.

\section{Animals}

Male Sprague-Dawley rats (Samtaco CO., Osan, Korea) were used throughout. Animals were acclimatized for 1 week before the experiments and housed in an airconditioned animal room under a 12-h light/dark cycle (08:00-20:00 h light, 20:00-08:00 h dark), at $23 \pm 5{ }^{\circ} \mathrm{C}$ and $55 \pm 10 \% \mathrm{RH}$ and provided a laboratory diet and water ad libitum. The experimental procedures were carried out according to the animal care guidelines of the NIH and the Ewha University Institutional Animal Care and Use Committee.

\section{Compound Synthesis}

JC3 was synthesized as described previously [18]. 4Hydroxy-3-methoxy cinnamaldehyde was protected with tert-butyldimethylsilyl trifluoromethanesulfonate in the presence of 2,6-lutidine or 2-(trimethylsilyl)ethoxymethyl chloride (SEM-Cl) $/ N, N$-di-isopropylehylamine to form aldehydes at yields of 95 and $97 \%$, respectively. JC3 was identified by infrared and NMR spectroscopy and highresolution mass spectroscopy [18].

\section{Experimental Paw Hyperalgesia and Arthritis Groups}

For the paw hyperalgesia model, rats were divided into four groups: the non-treated normal group (the NOR group; $n=5$ ), the carrageenan-induced control group (the CON group; $n=5$ ), the CON group administered $5 \mathrm{mg} / \mathrm{kg}$ of JC3 (the CON+JC3_5 group; $n=5$ ), and the CON group administered $10 \mathrm{mg} / \mathrm{kg}$ of JC3 (the $\mathrm{CON}+\mathrm{JC} 3 \_10$ group; $n=5$ ). For the arthritis model, rats were divided into four groups: the non-treated group (the NOR group; $n=5$ ), the carrageenan/kaolin-induced control group (the ART group; $n=5$ ), the ART group administered $5 \mathrm{mg} / \mathrm{kg}$ of JC3 (the ART+JC3 5 group; $n=5$ ), and the ART group administered $10 \mathrm{mg} / \mathrm{kg}$ of JC3 treatment (the ART+JC3_10 group; $n=5$ ).

\section{Evaluation of Paw Hyperalgesia}

To induce paw hyperalgesia, rats were administered an intra-plantar injection of $1 \%$ carrageenan $(0.1 \mathrm{~mL})$ in the posterior left paw, as described previously [25]. Three hours after injection, pain thresholds were measured using the Randall-Selitto test and a paw pressure analgesia instrument (Ugo Basile Biological Research Apparatus Co., Comeria-Varese, Italy). Ten rats were studied per group and the test was 
performed blind. Rats were starved overnight and JC3 was evaluated at doses of 5 and $10 \mathrm{mg} / \mathrm{kg}$. JC3 was injected $1 \mathrm{~h}$ before carrageenan injection. To evaluate paw hyperalgesia, we measured the tolerance to increasing mild pressure on the affected paw between a flat surface and a blunt pointer of the instrument, according to the manufacturer's instructions.

\section{Induction of Arthritis}

The 5\% carrageenan/kaolin-induced arthritic rat model was prepared as described previously [10]. Animals were briefly anesthetized with $3 \%$ isoflurane in a $\mathrm{N}_{2} \mathrm{O} / \mathrm{O}_{2}$ mixture. Arthritic inflammation was induced by a single injection of $3 \%$ carrageenan/kaolin suspended in $100 \mu \mathrm{L}$ of pyrogen-free sterile saline, into left knee joints. To evaluate the arthritic progression of carrageenan/kaolin-induced arthritis rat, three different parameters were measured: knee thickness, weight distribution ratio (WDR), and using knee flexion test squeaking scores. These were considered behavioral indicators of carrageenan/kaolin-induced arthritis through checking for 6 days. As arthritis progression, redness and swelling of knee joints and arthritic pain started to appear and reached a maximum on day 1 after the carrageenan/kaolin injection. Daily JC3 injections 5 or $10 \mathrm{mg} / \mathrm{kg}$ were started $24 \mathrm{~h}$ after the carrageenan/kaolin injection and continued for 5 days. All behavioral tests were performed blinded.

\section{Evaluation of Knee Thickness}

Thicknesses of both hind knees were measured daily using a dial thickness gauge for 6 days after carrageenan/kaolin injection, and mean values were recorded. Thicknesses are expressed as ratios versus thicknesses measured prior to carrageenan/kaolin injections.

\section{WDRs}

The WDR is a ratio of the percent of weight carried on each hind leg in which the weight-bearing forces of both hind limbs were measured with an incapacitance meter (Ugo Basile Biological Research Apparatus Co., Comeria-Varese, Italy), as previously described by Hwang et al. [10]. To evaluate arthritic pain, a rat was placed in the test box of an incapacitance meter, which contained a slanted plank is located. The force borne by each hind limb was measured using two mechanotransducers, separately placed below hind limbs. Weights borne by hind limbs were estimated using 5-s averages, and mean weights of four separate estimations were calculated. WDR \% was calculated using $100 \times$ (weight borne by ipsilateral limb/total weight borne by both limbs). The WDR of hind paws in the normal group was 50:50, indicating that $50 \%$ of the weight was carried in each hind paw. As ankle pain and swelling progressed due to arthritis, this balance moved in favor of contralateral limbs.

\section{Squeaking Test}

Arthritis-induced hyperalgesia was assessed by quantifying the total number of vocalizations evoked by knee flexion or extension. Squeaking scores derived using the modified method of Yu et al. were used to assess knee rigidity and pain while knee joints of hind limbs were gently flexed and extended [26]. The numbers of vocalizations emitted during flexion and extension periods were counted. The cyclic procedure consisting of five 5-s flexion and extension cycles per hind limb. A vocalization rating of 0 (no vocalization) or 1 (vocalization) was given based on response to flexion or extension. Thus, vocalization rating scores lay between 0 and 10 (maximum) for each hind limb. The squeaking test was performed daily for 6 days.

\section{ELISA}

Rat serum samples was obtained by centrifugation at $6500 \mathrm{rpm}$ for $20 \mathrm{~min}$ and stored at $-70{ }^{\circ} \mathrm{C}$ until use. Levels of the inflammatory mediators IL-6, TNF- $\alpha$, PGE2, and VEGF were measured using enzyme-linked immunosorbent assay (ELISA) kits (BD Biosciences Pharmingen, San Diego, CA, USA, for IL-6, and TNF- $\alpha$; R\&D Systems, Minneapolis, MN, USA, for PGE2; and Abcam, Cambridge, UK, for VEGF) according to the manufacturer's instructions as described previously [1]. For the in vitro study, triplicate cultures of FLSs were treated with various concentrations of JC3 $(1,5$, and $10 \mu \mathrm{g} / \mathrm{mL})$ and/or IL-1 $\beta$ (10 ng/mL; ProSpec, Rehovot, Israel) and then cultured for $24 \mathrm{~h}$. Supernatants were collected, centrifuged, and analyzed for IL-6, IL-8, and PGE2 expression using commercial ELISA kits, as described previously [1]. 


\section{Histological Assessment}

For hematoxylin-eosin histochemistry, knee tissues were fixed in $10 \%$ paraformaldehyde overnight, dehydrated in $99 \%$ ethanol, embedded in paraffin, sectioned at $6 \mu \mathrm{m}$ (Finesse 325; Thermo Shandon Co., UK), and mounted on slides. Before staining, sections on slides were deparaffinized. To investigate morphologic changes and eosinophil infiltration, sections were stained with hematoxylin (Merck, Darmstadt, Germany) and $1 \%$ eosin (Sigma-Aldrich Co., MO, USA), airdried, and cover-slipped. All slides $(\times 100$ magnification) were observed and photographed using a microscope equipped with camera (BX51; Olympus Ltd., Tokyo, Japan), and images were analyzed using DP2BSW software (Olympus Ltd., Tokyo, Japan). Subsequently, the stained sections were scored quantitatively in a blinded manner by three independent observers. Histology scores of extent of cellular infiltration and joint integrity were evaluated as follows: $0=$ normal; $1=$ infiltration of inflammatory cells; $2=2$ cartilage erosion and pannus formation; $3=$ bone erosion; and $4=$ bone destruction. The maximum score was 4 per mouse.

\section{Statistical Analysis}

Results are expressed as means \pm SEMs. The analysis was conducted by one-way ANOVA using SPSS Ver. 13.0 (SPSS; Chicago, USA). Statistical differences between groups were further analyzed using Tukey's post hoc test. $P$ values of $<0.05$ were considered statistically significant.

\section{RESULTS}

\section{Analgesic Effect of JC3 on Carrageenan-Induced Paw Hyperalgesia}

Treatment with JC3 at $1 \mathrm{~h}$ before carrageenan injection increased paw withdrawal thresholds versus those observed in the CON group (Fig. 1), indicating JC3 pretreatment inhibited carrageenan-induced hyperalgesia. Furthermore, paw withdrawal thresholds were significantly greater in the $\mathrm{CON}+\mathrm{JC} 3$ - 10 group than in the CON group.

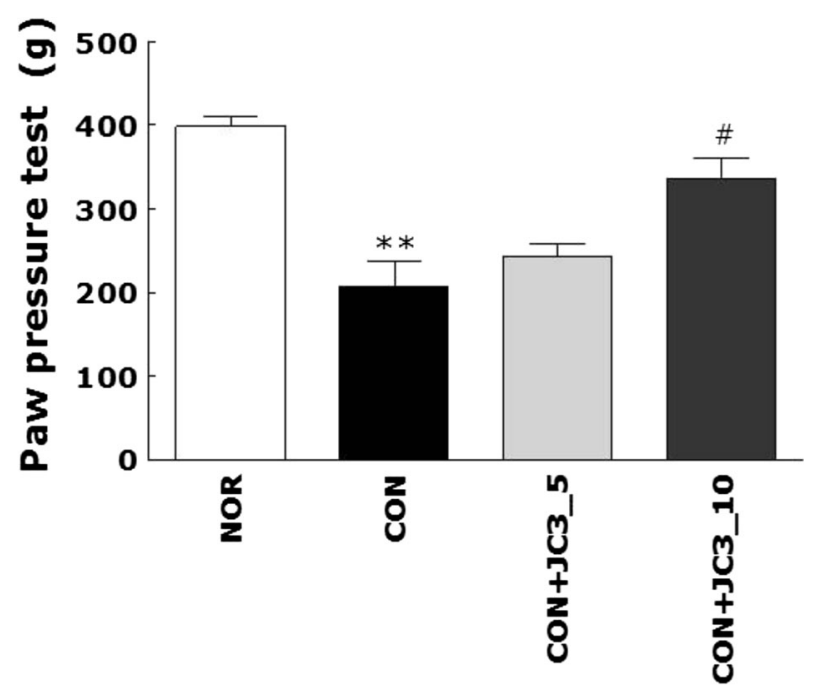

Fig. 1. Analgesic effect of JC3 in the carrageenan-induced hyperalgesia rat model. Hyperalgesia was induced by an intra-plantar injection of carrageenan $(100 \mu \mathrm{L}$ of $1 \%$ solution) into left hind paws. JC3 was administered (i.p.) $1 \mathrm{~h}$ before carrageenan injection. Nociceptive thresholds were determined by the Randall-Selitto test using a paw-pressure analgesy meter $3 \mathrm{~h}$ after carrageenan injection. NOR non-treated group, $\mathrm{CON}$ carrageenan-treated control group, $\mathrm{CON}+\mathrm{JC} 3$ - 5 carrageenaninduced and $5 \mathrm{mg} / \mathrm{kg} \mathrm{JC} 3$-treated groups, and $\mathrm{CON}+\mathrm{JC} 3 \_10$ carrageenan-induced and $10 \mathrm{mg} / \mathrm{kg} \mathrm{JC3}$-treated group. Results are presented as means \pm standard errors. Data analysis was performed using oneway ANOVA followed by Tukey's post hoc test. ${ }^{* *} p<0.01$ vs. NOR group, $\# p<0.05$ vs. CON group.

\section{Anti-Arthritic Effect of JC3 on Carrageenan/Kaolin- Induced Arthritis}

To examine the in vivo anti-arthritic effect of JC3, the efficacy of JC3 was evaluated by assessing physical parameters, such as knee morphology, knee thickness, WDR, and squeaking scores. In addition, morphological changes in tissues and inflammatory cell infiltration were also assessed histologically. The ART group exhibited signs of severe inflammation, such as synovial hyperplasia, pannus formation, bone erosion, and cartilage degradation, but these phenomena were inhibited by JC3 treatment (Fig. 2). Knee thickness was used as an indicator of arthritis induced by carrageenan/kaolin treatment, and the ART+JC3_10 group had significantly smaller knee thicknesses than the ART group (Fig. 3a). This result was evidenced by redness and swelling of the entire paw before reaching a plateau, which was steadily maintained throughout the experimental period. Next, we examined the effect of JC3 on WDRs of hind paws of rats with carrageenan/kaolin-induced arthritis (Fig. 3b). At baseline before carrageenan/kaolin injection, mean WDR 


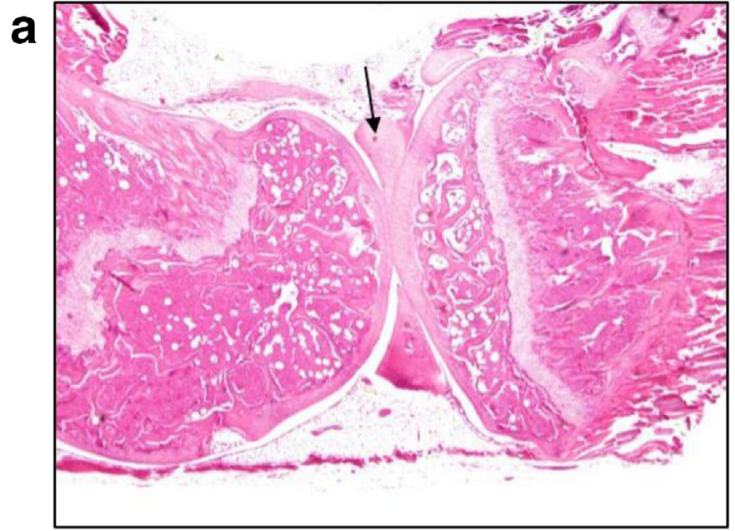

NOR

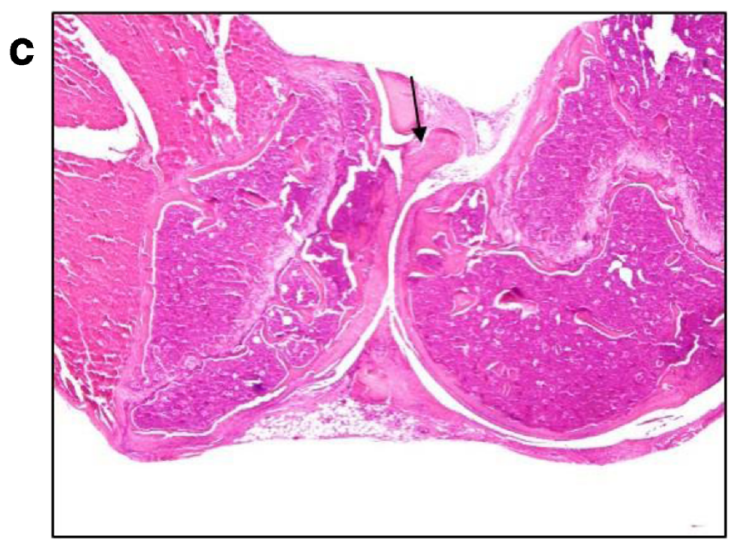

ART+JC3_5

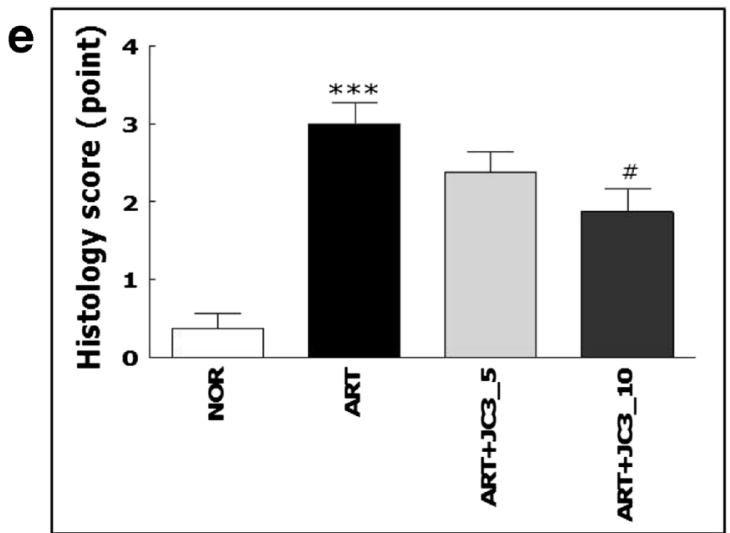

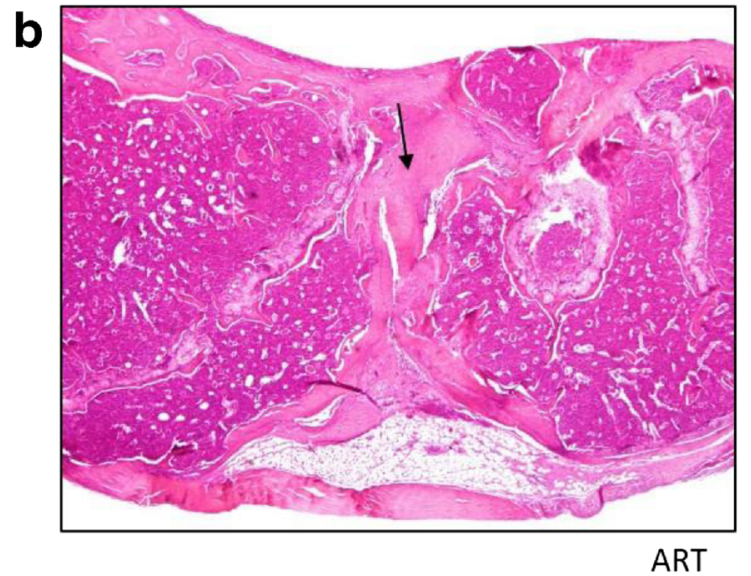

d

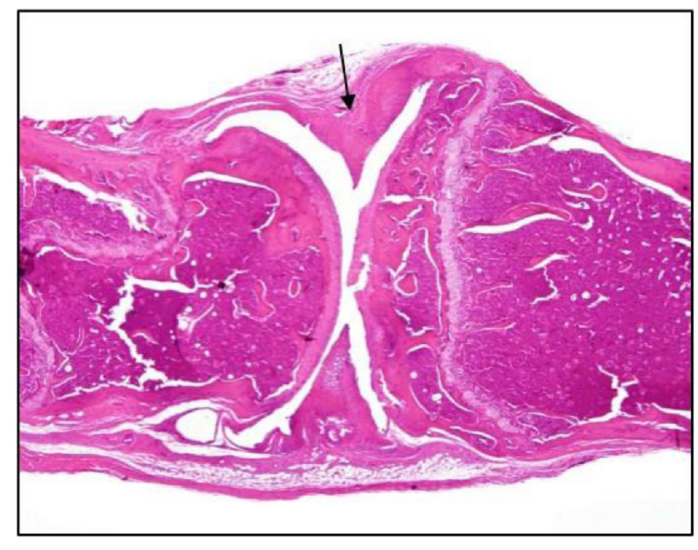

ART+JC3_10

Fig. 2. Histological images of the knee joints of rat administered JC3 in the NOR (a), ART (b), ART+JC3_5 (c), and ART+JC3_10 (d) groups. e Histology scores were determined using a standardized scoring scale as described in "MATERIALS AND METHODS." Tissues were stained with hematoxylin and eosin (HE). Results are presented as means \pm standard errors. $* * * p<0.005$ vs. the NOR group and $\# p<0.05$ vs. the ART group.

was 50:50. However, significant changes in WDR were observed on day 1 after carrageenan/kaolin injection, and on day 6 , the weight carried by ipsilateral damaged legs in the ART group reached $21 \%$. However, WDR did not deteriorate as much in animals administered 5 or $10 \mathrm{mg} / \mathrm{kg}$ $\mathrm{JC} 3$, and this effect was significant in the ART+JC3 10 . 

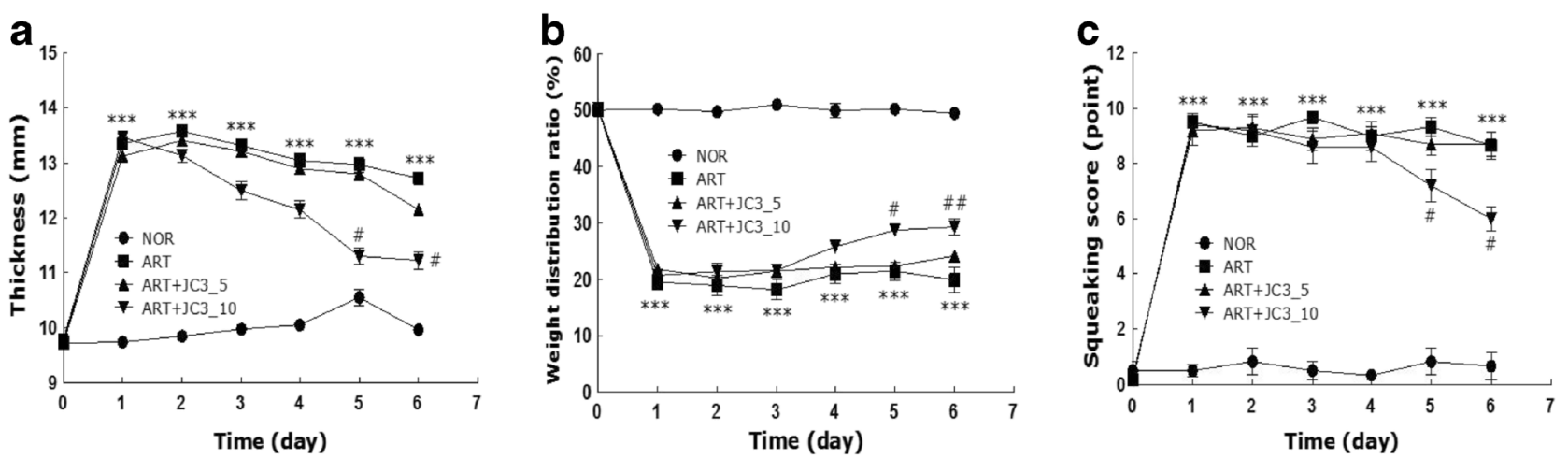

Fig. 3. Assessments of anti-arthritic activity in the rat model of carrageenan/kaolin-induced arthritis. a Thicknesses of knees, $\mathbf{b}$ weight distribution ratios (WDRs), and $\mathbf{c}$ squeaking scores, indicating arthritis severity. Results are presented as means \pm standard errors. $* * * p<0.005 v s$. the NOR group and $\# p<0.05$ and \#\#p<0.01 vs. the ART group.

Vocalization scores for extension and flexion of reached a maximum at day 1 after carrageenan/kaolin injection. In the ART+JC3 10 group, numbers of vocalizations were significantly less at 5 days after injection, whereas little analgesic effect was observed in the ART+JC3_5 group (Fig. 3c).

\section{Effect of JC3 on Inflammatory Mediators and}

\section{Angiogenic Mediator in the Serum of Carrageenan/}

\section{Kaolin-Administered Rats}

Carrageenan/kaolin injection significantly increased levels of the inflammatory mediators TNF- $\alpha$, IL-6, and PGE2 and the angiogenic mediator VEGF in serum. An increase in each mediator is an important component of arthritis. Thus, we investigated whether JC3 decreased each mediators in carrageenan/kaolininduced arthritis rats. A significant increase in serum TNF- $\alpha$, IL-6, PGE2, and VEGF level was observed in the ART group compared to the NOR group. Expectedly, JC3 was inhibited in TNF- $\alpha$, IL-6, PGE2, and VEGF level (Fig. 4).

\section{Effect of JC3 on the Productions of Inflammatory and}

\section{Angiogenic Mediators in FLSs}

FLSs were treated with IL- $1 \beta$ at $10 \mathrm{ng} / \mathrm{mL}$ with or without JC3 $(1,5$, and $10 \mu \mathrm{g} / \mathrm{mL})$. JC3 with or without IL- $1 \beta$ for $24 \mathrm{~h}$ did not affect FLS viability as compared with non-treated controls (data not shown). The overexpressions of PGE2, IL-6, and IL- 8 in synovium are well known to increase joint destruction and synovial inflammation. Thus, we examined whether JC3 could reduce the IL- $1 \beta$-induced overexpressions of PGE2, IL-6, and IL-8 in FLSs. Treatment with IL-1 $\beta$ significantly increased PGE2 and IL-6 levels as compared with controls. However, the addition of JC3 inhibited the up-regulations of PGE2 and IL-6 by IL$1 \beta$ (Fig. 5a, b). In a previous study, angiogenic activities in the conditioned media of inflamed human rheumatoid synovial tissue macrophages and of lipopolysaccharidestimulated blood monocytes were equally blocked by IL-8 antibody, and an IL-8 antisense oligonucleotide specifically blocked the production of monocyteinduced angiogenic activity. These observations show IL-8 is an important angiogenic factor of the pathogenesis of arthritis. When we examined the effect of JC3, we found it inhibited IL- $1 \beta$-induced IL- 8 secretion in FLSs (Fig. 5c), which suggested JC3 inhibits neovascularization in the inflamed joints of arthritic rats.

\section{DISCUSSION}

Damaging inflammation is the hallmark of RA, and it has become progressively clear even non-inflammatory arthritis is characterized by many progressive immunologically related events. In fact, consequent inflammation in synovial membranes and joint fluid are important symptoms of osteoarthritis, and eventually result in synovitis and synovial hyperplasia. Synovitis is a symptom of inflammatory and degenerative arthritis, and in RA, synovitis 

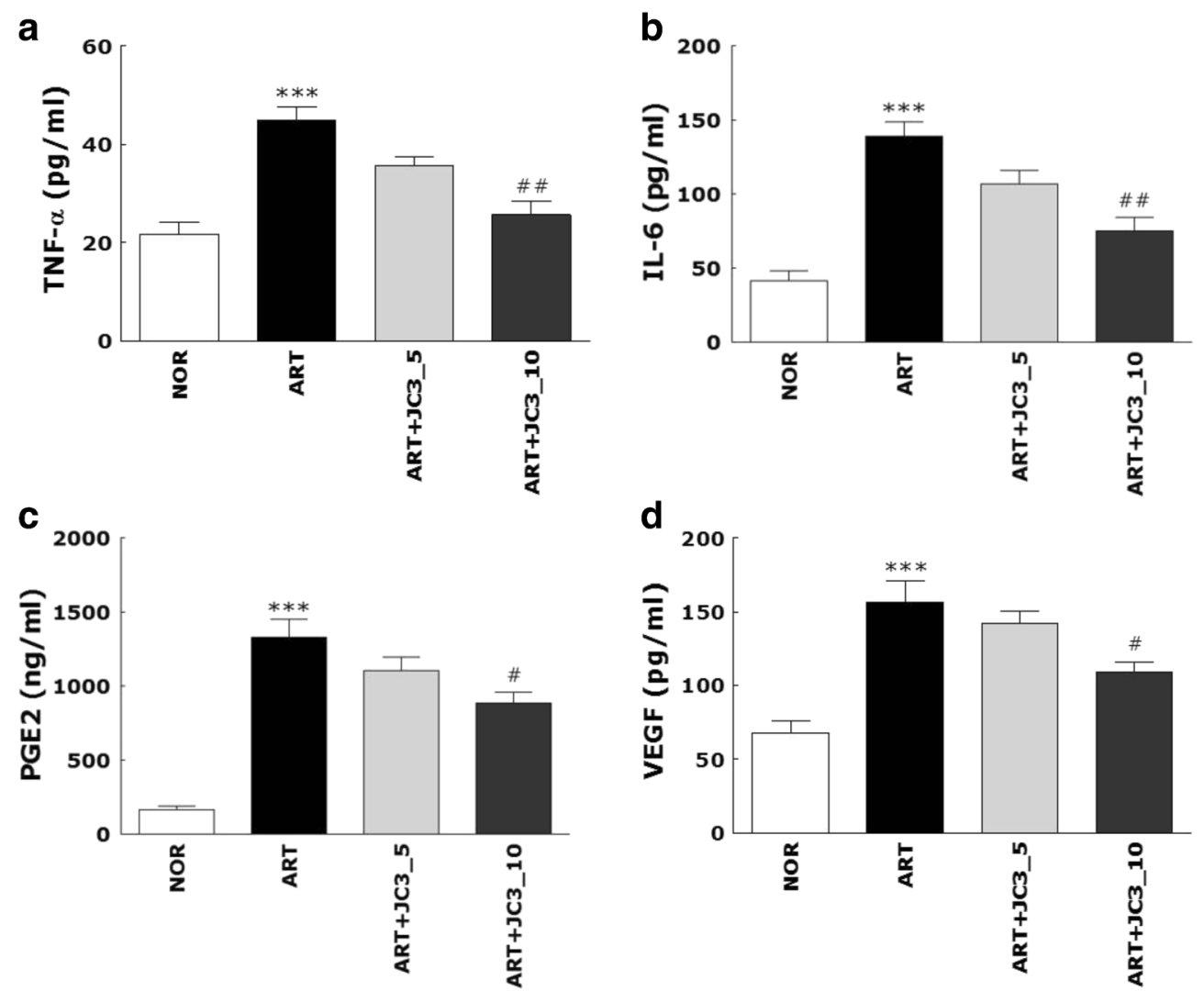

Fig. 4. Effects of JC3 on the ELISA determined levels of the pro-inflammatory mediators TNF- $\alpha$ (a), IL-6 (b), PGE2 (c), and VEGF (d) in serum of the rat model of carrageenan/kaolin-induced arthritis. Results are presented as means \pm standard errors. $* * * p<0.005 v s$. the NOR group and $\# p<0.05$ and $\# p<0.01$ vs. the ART group.

and synovial fibroblast activation are key stages in the development of invasive rheumatoid pannus [9].

Synovitis is usually accompanied by proliferation of synovial lining and resultant erosion of bone and joint cartilage. Macrophage-like synoviocytes (MLSs) and FLSs in inflamed synovium play significant roles in the destructive process of joint arthritis. FLSs in inflamed joints secrete inflammatory cytokines, such as IL-6 and IL-8. Furthermore, TNF- $\alpha$ produced by macrophages and FLSs infiltrates synovial linings and
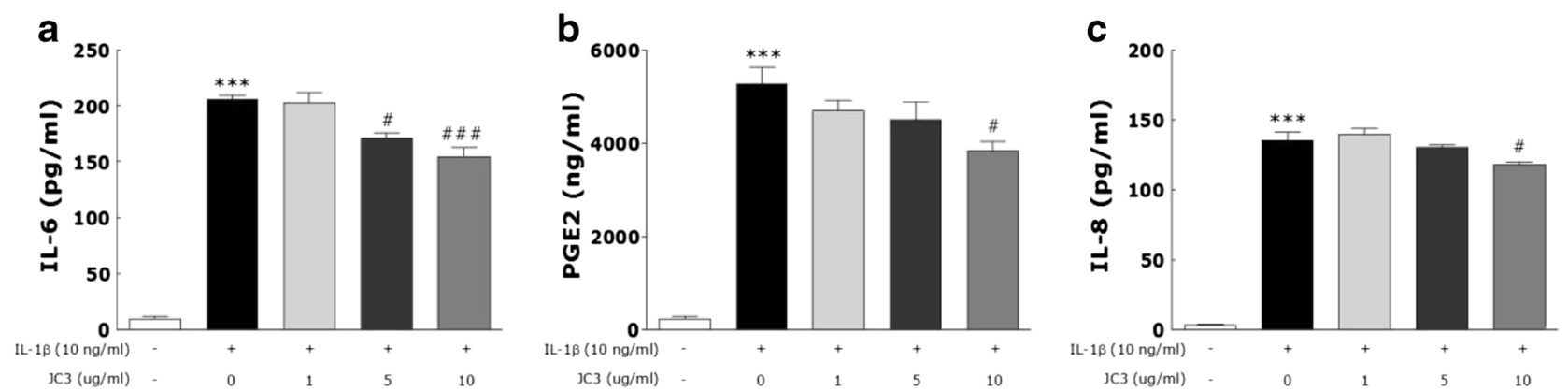

Fig. 5. Effects of JC3 on the ELISA determined levels of the pro-inflammatory mediators IL-6 (a) and PGE2 (b), and on the levels of IL-8 (angiogenic mediator) (c) in FLS cells. Results are presented as means \pm standard errors. $* * * p<0.001 v s$. nontreated controls, \#p $<0.05$ and \#\#\#p ${ }^{*} 0.005 v s$. IL-1 $\beta$ treated cells. 
contribute to the initiation of joint damage by upregulating the expressions of various hydrolytic enzymes and pro-inflammatory mediators.

Because of the etiological complexity and diversity of pathological mechanisms in inflammatory arthritis, many researchers have studied non-steroidal anti-inflammatory agents derived from natural sources with the aim of developing new clinical treatments [4]. Yakuchinone B is a constituent of the seeds of Alpina oxyphylla, a member of the ginger family (Zingiberaceae), and is commonly used in folk medicine. Yakuchinone B has a variety of biological effects, which include anti-inflammatory activity [22], as demonstrated by its strong inhibitory effect on prostaglandin biosynthesis in vitro [15]. In a previous in vitro study, it was shown JC3, a synthesized benzylideneacetophenone, has free radical scavenging activity and suppresses lipopolysaccharide (LPS)-induced nitric oxide (NO) generation and anti-excitotoxicity in cortical culture neurons [18]. Based on these results, we assessed the anti-arthritic effects of JC3 to determine whether it had therapeutic potential for the treatment of arthritis.

In this study, we used two animal models to evaluate the in vivo analgesic and anti-arthritic effect of JC3. First, we found JC3 significantly ameliorated pain the CON+JC3 10 group in our carrageenan-rat model.

Second, JC3 seemed to inhibit the development of arthritic symptoms as based on knee thicknesses, WDRs, squeaking scores, and ELISA determined TNF- $\alpha$, IL-6, and PGE2 levels in serum. In the case of knee thicknesses, which were used to assess degree of edema caused by arthritic inflammation, mean knee thickness in the ART+JC3_10 group was less than in the ART group from day $\overline{3}$, and significantly less on day 6. Furthermore, before arthritis induction, mean WDR were 50:50, but from 1 day after carrageenan/ kaolin injection, WDR rapidly changed. In the JC3 treatment groups, during the experimental period (6 days), the ratio was continuously balanced. The efficacy of the ART+JC3_10 group was observed after day 4. For these reasons, the efficacy of the JC3 treatment in terms of WDR recovery was significantly verified in this study. In addition, squeaking scores in ART+JC3_10 group were lower than in ART group from day $\overline{4}$, and significantly lower on day 5 and 6 .

Next, we found JC3 significantly inhibited the productions of three important proinflammatory mediators, TNF- $\alpha$, IL-6, and $\mathrm{PGE}_{2}$, and inhibited the angiogenic mediator, IL-8, in carrageenan/kaolin-induced rats and IL-1 $\beta$-stimulated human FLSs. This inhibition of $\mathrm{PGE}_{2}$ production is important because $\mathrm{PGE}_{2}$ is a known key mediator of inflammatory pain [7]. We also investigated the effect of JC3 on IL-8 (a mediator of angiogenesis) [20] and found that its expression was in the inflammed joints of arthritic rats was inhibited by JC3 treatment.

Finally, the pathological angiogenesis that leads to worsening of arthritis is the critical process [19]. The development of such morbid blood vessel increases the amount of nutrients going into the synovial tissue, increases the movement of immune, and inflammatory cells from the joint, where the inflammation occurred. Due to this result, it becomes the strong provider of the inflammatory cytokine, chemokine, and the growth factor, which then leads to worsening of arthritis [23]. Therefore, when the angiogenesis is inhibited, it inhibits the abnormal growth of inflammation and synovial tissue, and leads to the treatment of arthritis. This study has confirmed decrease in secretion of VEGF of an arthritic animal model with JC3 treatment. Therefore, it is safe to think that this result is shown to have an effect in the treatment of arthritis with JC3.

We reported that JC3 has anti-arthritic effects in animal models and anti-inflammatory effects in IL- $1 \beta$ stimulated FLSs. As regards the arthritic model used, we found the carrageenan/kaolin injection into the knee joint produced arthritic symptoms, such as edema, pain, stiffness, and rigidity. Collectively, our results suggest that JC3 could be considered as a potential therapeutic agent for the treatment of arthritis.

\section{ACKNOWLEDGEMENTS}

This research was supported by a National Research Foundation (NRF) grant funded by the Korean Ministry of Science, ICT \& Future Planning (MRC 2010-0029355).

\section{COMPLIANCE WITH ETHICAL STANDARDS}

The experimental procedures were carried out according to the animal care guidelines of the NIH and the Ewha University Institutional Animal Care and Use Committee.

Open Access This article is distributed under the terms of the Creative Commons Attribution 4.0 International License (http://creativecommons.org/licenses/by/4.0/), which permits unrestricted use, distribution, and reproduction in any medium, provided you give appropriate credit to the original author(s) and the source, provide a link to the Creative Commons license, and indicate if changes were made. 
Publisher's Note Springer nature remains neutral with regard to jurisdictional claims in published maps and institutional affiliations.

\section{REFERENCES}

1. Bang, J.S., D.H. Oh, H.M. Choi, B.J. Sur, S.J. Lim, J.Y. Kim, H.I. Yang, M.C. Yoo, D.H. Hahm, and K.S. Kim. 2009. Antiinflammatory and antiarthritic effects of piperine in human interleukin 1beta-stimulated fibroblast-like synoviocytes and in rat arthritis models. Arthritis Research and Therapy 11 (2): R49. https://doi.org/ $10.1186 /$ ar2662

2. Bar-Yehuda, S., L. Rath-Wolfson, L. Del Valle, A. Ochaion, S. Cohen, R. Patoka, G. Zozulya, F. Barer, E. Atar, S. Piña-Oviedo, G. Perez-Liz, D. Castel, and P. Fishman. 2009. Induction of an antiinflammatory effect and prevention of cartilage damage in rat knee osteoarthritis by CF101 treatment. Arthritis and Rheumatism 60 (10): 3061-3071. https://doi.org/10.1002/art.24817.

3. Braddock, M., A. Quinn, and J. Canvin. 2004. Therapeutic potential of targeting IL-1 and IL-18 in inflammation. Expert Opinion on Biological Therapy 4 (6): 847-860. https://doi.org/10.1517/ 14712598.4.6.847.

4. Chrubasik, J.E., B.D. Roufogalis, and S. Chrubasik. 2007. Evidence of effectiveness of herbal antiinflammatory drugs in the treatment of painful osteoarthritis and chronic low back pain. Phytotherapy Research 21 (7): 675-683. https://doi.org/10.1002/ptr.2142.

5. Chun, K.S., Y. Sohn, H.S. Kim, O.H. Kim, K.K. Park, J.M. Lee, A. Moon, S.S. Lee, and Y.J. Surh. 1999. Anti-tumor promoting potential of naturally occurring diarylheptanoids structurally related to curcumin. Mutation Research 428 (1-2): 49-57. https://doi.org/ 10.1016/S1383-5742(99)00031-9.

6. Dayer, J.M., B. de Rochemonteix, B. Burrus, S. Demczuk, and C.A. Dinarello. 1986. Human recombinant interleukin 1 stimulates collagenase and prostaglandin E2 production by human synovial cells. The Journal of Clinical Investigation 77 (2): 645-648. https:// doi.org/10.1172/JCI112350.

7. Dray, A. 1995. Inflammatory mediators of pain. British Journal of Anaesthesia 75 (2): 125-131. https://doi.org/10.1093/bja/75.2.125.

8. Gaby, A.R. 1999. Alternative treatments for rheumatoid arthritis Alternative Medicine Review 4 (6): 392-402.

9. Han, J., W. Wang, C. Qu, R. Liu, W. Li, Z. Gao, and X. Guo. 2015. Role of inflammation in the process of clinical Kashin-Beck disease: Latest findings and interpretations. Inflammation Research 64 (11): 853-860. https://doi.org/10.1007/s00011-015-0861-6.

10. Hwang, H.J., H.J. Lee, C.J. Kim, I. Shim, and D.H. Hahm. 2008 Inhibitory effect of amygdalin on lipopolysaccharide-inducible TNF-alpha and IL-1beta mRNA expression and carrageenaninduced rat arthritis. Journal of Microbiology and Biotechnology 18 (10): 1641-1647.

11. Itoh, F., S. Aoyagi, H. Kusama, M. Kojima, and H. Kogo. 2004. Effects of clodronate and alendronate on local and systemic changes in bone metabolism in rats with adjuvant arthritis. Inflammation 28 (1): 15-21 https://doi.org/0360-3997/04/0200-0015/0.

12. Jacobs, J.W.G., F.W. Kraaimaat, and J.W. Bijlsma. 2001. Why do patients with rheumatoid arthritis use alternative treatments? Clinical
Rheumatology 20 (3): 192-196. https://doi.org/10.1007/ PL00011199.

13. Kay, J., and L. Calabrese. 2004. The role of interleukin-1 in the pathogenesis of rheumatoid arthritis. Rheumatology 43 (3): iii2-iii9. https://doi.org/10.1093/rheumatology/keh201.

14. Kim, K.S., E.K. Park, S.M. Ju, H.S. Jung, J.S. Bang, C. Kim, Y.A. Lee, S.J. Hong, S.H. Lee, H.I. Yang, and M.C. Yoo. 2007. Taurine chloramine differentially inhibits matrix metalloproteinase 1 and 13 synthesis in interleukin-1beta stimulated fibroblast-like synoviocytes. Arthritis Research and Therapy 9 (4): R80. https:// doi.org/10.1186/ar2279.

15. Kiuchi, F., S. Iwakami, M. Shibuya, F. Hanaoka, and U. Sankawa. 1992. Inhibition of prostaglandin and leukotriene biosynthesis by gingerols and diarylheptanoids. Chemical and Pharmaceutical Bulletin 40 (2): 387-391. https://doi.org/10.1248/cpb.40.387.

16. Mor, A., S.B. Abramson, and M.H. Pillinger. 2005. The fibroblastlike synovial cell in rheumatoid arthritis: A key player in inflammation and joint destruction. Clinical Immunology 115 (2): 118-128. https://doi.org/10.1016/j.clim.2004.12.009.

17. Ninomiya, Y., N. Shimma, and H. Ishitsuka. 1990. Comparative studies on the antirhinovirus activity and the mode of action of the rhinovirus capsid binding agents, chalcone amides. Antiviral Research 13 (2): 61-74. https://doi.org/10.1016/01663542(90)90022-Y.

18. Oh, S., S. Jang, D. Kim, I.O. Han, and J.C. Jung. 2006. Synthesis and evaluation of biological properties of benzylideneacetophenone derivatives. Archives of Pharmacal Research 29 (6): 469-475. https://doi.org/10.1007/BF02969418.

19. Paleolog, E.M. 2002. Angiogenesis in rheumatoid arthritis. Arthritis Research 4 (3): S81-S90. https://doi.org/10.1186/ar575.

20. Panayi, G.S. 1997. T-cell-dependent pathways in rheumatoid arthritis. Current Opinion in Rheumatology 9 (3): 236-240.

21. Srimal, R.C., J.N. Sharma, A.N. Tangri, and B.N. Dhawan. 1973. Experimental evaluation of anti-inflammatory activity of 3,4dimethoxyphenylethylamino-3-aminopyridine (compound 64-92). Indian Journal of Experimental Biology 11 (3): 183-187.

22. Surh, Y.J. 2002. Anti-tumor promoting potential of selected spice ingredients with antioxidative and anti-inflammatory activities: A short review. Food and Chemical Toxicology 40 (8): 1091-1097. https://doi.org/10.1016/S0278-6915(02)00037-6.

23. Szekanecz, Z., and A.E. Koch. 2007. Mechanisms of disease: Angiogenesis in inflammatory diseases. Nature Clinical Practice Rheumatology 3 (11): 635-643. https://doi.org/10.1038/ncprheum0647.

24. Yamazaki, R., H. Hatano, R. Aiyama, T. Matsuzaki, S. Hashimoto, and T. Yokokura. 2000. Diarylheptanoids suppress expression of leukocyte adhesion molecules on human vascular endothelial cells. European Journal of Pharmacology 404 (3): 375-385. https:// doi.org/10.1016/S0014-2999(00)00620-8.

25. Yoo, E.A., S.D. Kim, W.M. Lee, H.J. Park, S.K. Kim, J.Y. Cho, W Min, and M.H. Rhee. 2008. Evaluation of antioxidant, antinociceptive, and anti-inflammatory activities of ethanol extracts from Aloe saponaria Haw. Phytotherapy Research 22 (10): 13891395. https://doi.org/10.1002/ptr.2514.

26. Yu, Y.C., S.T. Koo, C.H. Kim, Y. Lyu, J.J. Grady, and J.M. Chung. 2002. Two variables that can be used as pain indices in experimenta animal models of arthritis. Journal of Neuroscience Methods 115 (1): 107-113. https://doi.org/10.1016/S0165-0270(02)00011-0. 